\title{
Understanding Photovoltaic Cell Dynamic Resistance Behavior with Changing Incident Light Intensity
}

\author{
Francisco J. García-Sánchez, Senior Member, IEEE
}

\begin{abstract}
We present a theoretical examination of the general behavior that should be expected to be displayed by the magnitude of the dynamic resistance of a conventional illuminated photovoltaic device within the power-generating quadrant of its $I$ $V$ characteristics, when measured in quasi-static conditions from the short-circuit point to the open-circuit point, at various incident illumination intensities. The analysis is based on assuming that the photovoltaic device in question may be adequately described by a simple conventional d-c lumped-element single-diode equivalent circuit solar cell model, which includes significant constant series and shunt resistive losses, but lacks any other secondary effects. Using explicit analytic expressions for the dynamic resistance, we elucidate how its magnitude changes as a function of the terminal variables, the incident illumination intensity and the model's equivalent circuit elements' parameters.
\end{abstract}

Index Terms-Photovoltaic device, solar cell model, dynamic resistance, Lambert $W$ function.

\section{INTRODUCTION}

$\mathrm{T}$ HE negative value of the reciprocal slope of the $I-V$ characteristics of an illuminated photovoltaic cell is usually called its dynamic resistance, $r$. This is a very useful parameter to measure when analyzing and optimizing the cell's performance capabilities [1]. It is also essential when defining a solar cell's maximum power point (MPP). Here we will look, from a basic theoretical point of view, into some fundamental issues regarding the dynamic resistance of conventional photovoltaic cells, with the intention of better understanding its expected general behavior as incident light intensity changes. The questions to be addressed are:

1) Does the magnitude of the dynamic resistance $r$ of conventional photovoltaic cells depend on the intensity of the incident illumination? Or, equivalently, is $r$ dependent on the magnitude of the photo-current, Iph, produced by the separation and collection of photo-generated charge carriers?

2) Dos the magnitude of $r$ measured under short-circuit or open-circuit conditions depend on incident light intensity?

3) If so, how are these dependencies?

To obtain theoretical answers to the above fundamental queries, we will start from the mathematical equations of the

Manuscript originally written starting 18 November 2019. Date of current draft version 28 November 2019.

F. J. García-Sánchez is a Professor Emeritus of the Department of Electronics and Circuits of Universidad Simón Bolívar, Caracas, Venezuela. He

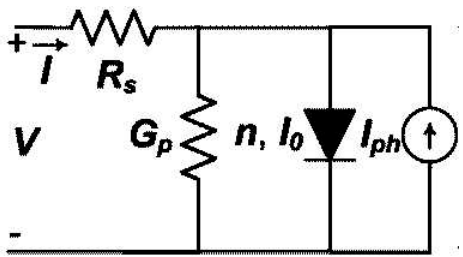

Fig. 1. Equivalent lumped-element dc circuit model used to describe the current-voltage characteristics at the terminals of a conventional illuminated cell.

simplest model that can be used to describe the most significant physical phenomena responsible for the typical shape of conventional photovoltaic cells' static $I-V$ characteristics under illumination. That simplest realistic model is an equivalent condensed-element (a.k.a. lumped-parameter) dc electric circuit [1], whose configuration is that shown in Fig. 1.

The electric elements of this circuit consist of a parallelconnected combination of: a single diode (characterized by the linked pair of lumped parameter values $n$ and $I_{0}$, which merge the most significant charge carrier transport mechanisms at the cell's junction); a photo-generated current source (with magnitude defined by its lumped parameter Iph) oriented in the direction of the diode's reverse current, which accounts for the photo-current produced by all collected photo-generated carriers; and a shunt resistor (specified by the value of its lumped parameter $R p=1 / G p$ ) that includes all resistive losses across the cell's junction. As shown in Fig. 1, this parallel combination is further connected in series to a second resistor (specified by the value of its lumped parameter $R s$ ), which combines all series resistive losses caused by the cell's contacts and terminals. Both resistive elements are assumed to stay approximately constant with respect to the circuit' variables and illumination intensity.

We will assume that the magnitude of the dynamic resistance, $r$, embodied by the value of the illuminated photovoltaic cell $I$ - $V$ characteristics' reciprocal slope, can be experimentally determined at quasi-static conditions, either by performing a small-signal ac measurement at very low frequency, or by numerical calculation from two or more consecutive data points taken around the location of interest on the measured static $I-V$ characteristic [2].

is also an Honorary Collaborator at the Superior School of Experimental Sciences and Technology of Universidad Rey Juan Carlos, Madrid, Spain, E.U. (e-mail: fgarcia@ieee.org). @2019 F. J. García-Sánchez. 


\section{METHODS}

The first step to theoretically study the behavior of the dynamic resistance is to write the equation that describes the simplest equivalent circuit model capable of adequately representing the conventional non-ideal photovoltaic cell with series and parallel resistive losses $(R s \neq 0, G p \neq 0)$. We have chosen to use the single-diode model whose lumped-element equivalent circuit [1] is shown in Fig. 1. The relationship between its terminals' current and voltage is mathematically expressed using Kirchoff's Laws and Shockley's diode equation [3], which results in the following implicit transcendental linear-exponential equation:

$$
I=I_{p h}-I_{0}\left[\exp \left(\frac{I R_{S}+V}{n v_{t h}}\right)-1\right]-\left(I R_{S}+V\right) G_{p} .
$$

Notice that in writing (1) we have defined the current as coming out of the positive terminal, that is, in the opposite direction (sign) as that shown in Fig. 1. We do so for convenience and without loss of generality, in order to adhere to the sign convention normally used by photovoltaic power engineers, where the power generating quadrant is presented as the first (instead of as the fourth) quadrant of the illuminated cell's $I$ - $V$ characteristics (see Fig. 2).

\section{A. Explicit solutions}

Closed-form solutions of the cell's terminal current and voltage equations as explicit functions of each other are preferred over (1) from simulation and analysis points of views. Such explicit solutions are possible to be obtained from (1) thanks to the use of the Lambert $W$ function [4]. The solution of (1) for the terminal current as an explicit function of the terminal voltage is [5]:

$$
I(V)=-\frac{n v_{t h}}{R_{S}} W_{0}\left\{\frac{I_{0} R_{S} \exp \left[\frac{\left(I_{0}+I_{p h}\right) R_{S}+V}{n v_{t h}\left(G_{p} R_{S}+1\right)}\right]}{n v_{t h}\left(G_{p} R_{S}+1\right)}\right\}-\frac{-\frac{\left(I_{0}+I_{p h}\right) R_{S}+V}{\left(G_{p} R_{S}+1\right)}+V}{R_{S}} .(2)
$$

where $W_{0}$ represents the principal branch of the Lambert $W$ function [4]. To find the corresponding expression for the terminal current, $I i$, of an ideal cell, i.e., a cell with negligible series and parallel resistive losses, substituting $R s=0$ and $G p=0$ directly into (2) will not work. Strictly, we would have to find the limit of (2) as Rs $\rightarrow 0$ and $\mathrm{Gp} \rightarrow 0$. However, a direct route is to simply substitute $R s=0$ and $G p=0$ into (1):

$$
I_{i}\left(V_{i}\right)=I_{p h}-I_{0}\left[\exp \left(\frac{V_{i}}{n v_{t h}}\right)-1\right] .
$$

Likewise, the solution of (1) for the terminal voltage as an explicit function of the terminal current can be written also in terms of the Lambert $W$ function [5]:

$$
V(I)=-n v_{t h} W_{0}\left[\frac{I_{0} \exp \left(\frac{I_{0}+I_{p h}-I}{G_{p} n v_{t h}}\right)}{G_{p} n v_{t h}}\right]+\frac{I_{0}+I_{p h}-I}{G_{p}}-I R_{S}
$$

The corresponding expression for the terminal voltage, $\mathrm{Vi}$, of the ideal cell is quickly found by inverting (2i):

$$
V_{i}\left(I_{i}\right)=n v_{t h} \ln \left(\frac{I_{p h}+I_{0}-I_{i}}{I_{0}}\right)
$$

\section{B. Short-circuit and open-circuit expressions}

An expression for the short circuit current Isc can be written by evaluating (1) at $V=0$ :

$$
I_{S c}=-\frac{n v_{t h}}{R_{S}} W_{0}\left[\frac{I_{0} R_{S} \exp \left(\frac{\left(I_{0}+I_{p h}\right) R_{S}}{n v_{t h}\left(G_{p} R_{S}+1\right)}\right)}{n v_{t h}\left(G_{p} R_{S}+1\right)}\right]+\frac{\left(I_{0}+I_{p h}\right)}{\left(G_{p} R_{S}+1\right)},
$$

To find the corresponding expression for the short circuit current, Iisc, of an ideal cell, substituting $R s=0$ and $G p=0$ directly into (4) will not work. Strictly, we would have to find the limit of (4) as $R s \rightarrow 0$ and $G p \rightarrow 0$. A much more direct route is to simply evaluate (2i) and at $V=0$ :

$$
I_{i s c}=I_{p h},
$$

The expression for the open circuit voltage $V o c$ is found by evaluating (2) at $I=0$ :

$$
V_{o c}=-n v_{t h} W_{0}\left[\frac{I_{0} \exp \left(\frac{I_{0}+I_{p h}}{G_{p} n v_{t h}}\right)}{G_{p} n v_{t h}}\right]+\frac{I_{0}+I_{p h}}{G_{p}} .
$$

Again, to find the corresponding expressions for the opencircuit voltage, Vioc, of an ideal cell, substituting $R s=0$ and $G p=0$ directly into (5) will not work. We would need to find the limit of (5) as $R s \rightarrow 0$ and $G p \rightarrow 0$. However, a simpler direct route is to evaluate (3i) and at $I=0$ :

$$
V_{i o c}=n v_{t h} \ln \left(\frac{I_{p h}+I_{0}}{I_{0}}\right) .
$$

The above equations clearly indicate, that the short-circuit current and the open-circuit voltage of an ideal or real photovoltaic cell are both functions of incident light intensity (represented by the value of the photo-current Iph). They also depend on the equivalent circuit's lumped elements parameter values, except that $V o c$ does not depend on the value of $R s$, since the voltage drop across $R s$ is zero when $I=0$.

\section{A hypothetical photovoltaic cell example}

We will use a hypothetical photovoltaic cell, with known equivalent circuit lumped-elements' parameter values, as an example to generate synthetic $I-V$ characteristics that will help to analyze and graphically illustrate the behavior of the dynamic resistance. The following parameters values of the elements of the circuit model shown in Fig. 1 will be used as example: diode's junction quality factor $n=1.5$, diode's reverse saturation 


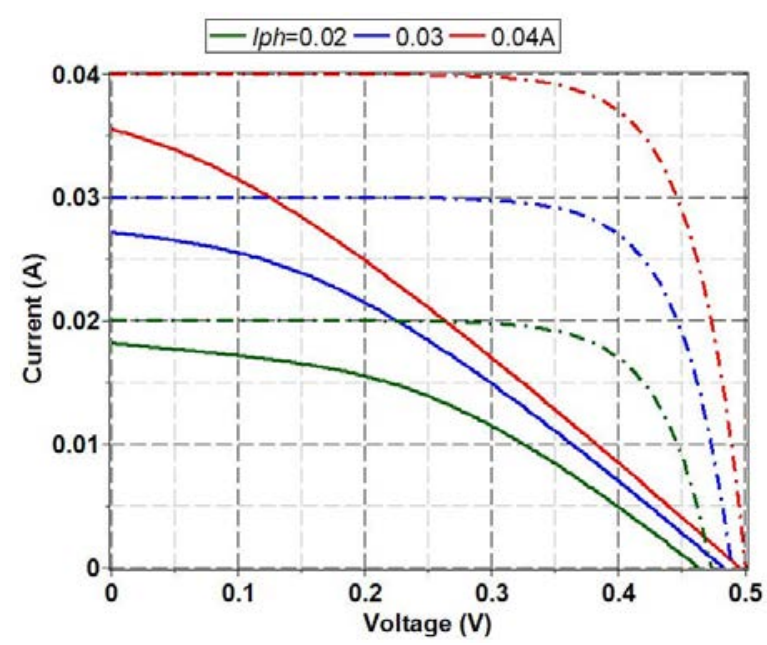

Fig. 2. Power-generating quadrant of synthetic current-voltage characteristics of a hypothetical illuminated real and ideal photovoltaic cell, with (solid lines) and without (dashed lines) resistive losses, respectively. The cell is modeled at room temperature by the equivalent circuit of Fig. 1, using the diode's parameters given in the text. Solid lines: $1 / G p=R p=100 \Omega$ and $R s=10 \Omega$; Dashed lines: $G p=0$ and $R s=0$ (ideal cell). Curves calculated for three indicated values of photo-current. The negative of the curves' reciprocal slope at any point is called the dynamic resistance.

current $I_{0}=10^{-7} \mathrm{~A}$, shunt resistive loss given by the stated value of $R p=1 / G p$, and series resistive loss given by the stated value of $R$ s. We assume room temperature operation, i.e. a thermal voltage $v_{t h}=\mathrm{k}_{\mathrm{B}} T / \mathrm{q}=0,02586 \mathrm{~V}$.

Three synthetic $I-V$ characteristics of this hypothetical cell, calculated using the explicit solution for the current given by (2), are presented in Fig. 2, for three intensities of incident light which generate within the cell corresponding photo-current magnitudes of $I p h=0.02,0.03$, and $0.04 \mathrm{~A}$. Matching $I-V$ curves of an ideal cell, alike but without shunt or series resistive losses $(R p=\mathrm{Gp}=0, R s=0)$, are also shown for visual comparison.

The numeric values of short circuit current and open circuit voltage, calculated with (4), (4i), (5) and (5i) for this particular example, at three given incident light intensities, are presented in Table I. They correspond to the axes intercepts of the three $I$ $V$ characteristics shown in Fig.2. It is worthwhile to point out that the sometimes hastily stated assumption that Isc $\approx I p h$ is not always a valid approximation, as (4) clearly implies. Its validity depends on the relative magnitudes of the cell's parallel and series resistive losses.

\section{RESULTS}

\section{A. Behavior of the dynamic resistance along the I-V curves}

The magnitude of the dynamic resistance, $r$, was already defined in Section I as the negative of the reciprocal slope of the synthetic $I-V$ characteristics. Explicit analytic expressions for $r$ as a function of the terminal current, and of its reciprocal the dynamic conductance, $g$, are obtained by taking the corresponding derivatives of the explicit solutions (2) and (1) with respect to current and voltage, respectively. This can be accomplished without difficulty because the Lambert $W$ function is readily differentiable [4]. The resulting explicit expressions for $r$ and $g$ are:
TABLE I

CALCULATED SHORT-CIRCUIT CURRENT AND OPEN-CIRCUIT VOLTAGE VALUES OF HYPOTHETICAL REAL AND IDEAL SOLAR CELLS AT THREE VALUES OF INCIDENT LIGHT INTENSITY

\begin{tabular}{|c|cc|cc}
\hline \hline \multirow{2}{*}{ Iph $(\mathrm{mA})$} & \multicolumn{2}{|c|}{ Isc $(\mathrm{mA})$} & \multicolumn{2}{c}{ Voc $(\mathrm{V})$} \\
\cline { 2 - 5 } & real & ideal & real & ideal \\
\hline 20 & 18.2 & 20 & 0.463 & 0.473 \\
30 & 27.2 & 30 & 0.482 & 0.489 \\
40 & 35.5 & 40 & 0.495 & 0.500 \\
\hline \hline
\end{tabular}

Equivalent circuit elements' parameters: $v_{t h}=0,02586 \mathrm{~V}, n=1.5, I_{0}=10^{-7} \mathrm{~A}$, Real: $R p=100 \Omega$, and $R s=10 \Omega$; Ideal: $G p=0$, and $R s=0$;

$$
r(I)=\frac{d V(I)}{d I}=-\frac{1}{G_{p}} \frac{1}{1+W_{0}\left[\frac{I_{0} \exp \left(\frac{I_{0}+I p h^{-I}}{G_{p} n v_{t h}}\right)}{G^{n} v_{t h}}\right]}-R_{S}
$$

and

$$
g(V)=\frac{d I(V)}{d V}=-\frac{W_{0}\left[\frac{I_{0} R_{S} \exp \left(\frac{\left(I_{0}+I_{p h}\right) R_{S}+V}{n v_{t h}\left(G_{p} R_{S}+1\right)}\right)}{n v_{t h}\left(G_{p} R_{S}+1\right)}\right]+\frac{G_{p} R_{S}}{\left(G_{p} R_{S}+1\right)}}{R_{S}\left\{1+W_{0}\left[\frac{I_{0} R_{S} \exp \left(\frac{\left(I_{0}+I_{p h}\right) R_{S}+V}{n v_{t h}\left(G_{p} R_{S}+1\right)}\right)}{n v_{t h}\left(G_{p} R_{S}+1\right)}\right]\right\}} .
$$

Fig. 3 presents the resulting evolution of the dynamic resistance $r$ as a function of voltage, calculated along the $I-V$ curve of the illustrative example cell, from the short-circuit point $(V=0)$ to the open circuit point $(V=V o c)$, at three levels of photo-current, Iph, intensity. Fig. 3 indicates that the magnitude of the dynamic resistance $r$, for any illumination level, decreases as the terminal voltage increases from the shortcircuit point to the open-circuit point. Alternative expressions of $r$ as a function of both the terminal voltage and current can be obtained by direct differentiation of the implicit (1):

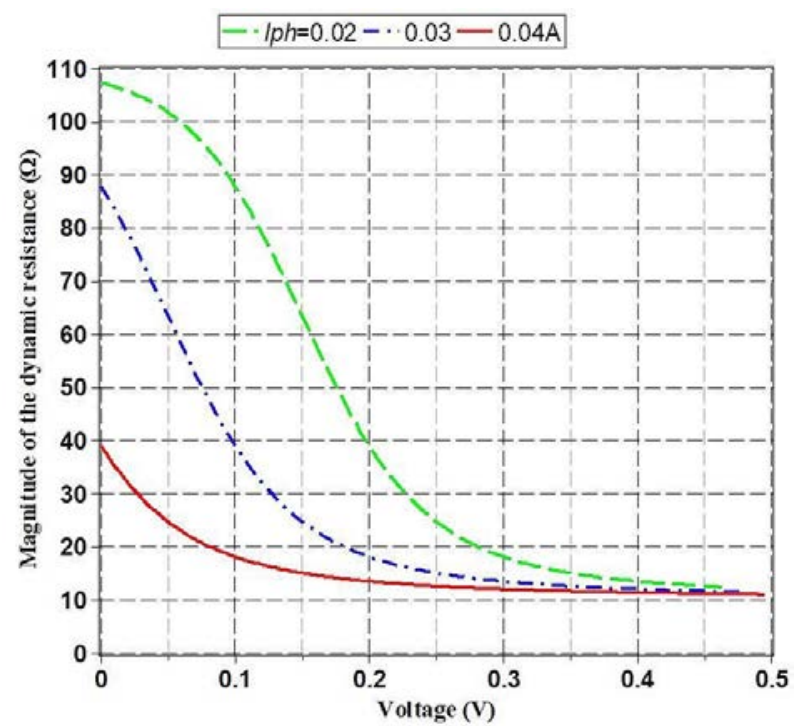

Fig. 3. Magnitude of the dynamic resistance $r$ of the hypothetical illuminated cell with $R p=100 \Omega$, and $R s=10 \Omega$, calculated with either (6) or (7) at three values of photo-current intensity Iph, from the short-circuit point $(V=0)$ to the open-circuit point $(V=V o c)$. These values of $r$ correspond to the negative reciprocals of the $I-V$ curves' slopes shown in Fig. 2. 


$$
1 / r(V, I)=\frac{d I}{d V}=-\left(1+\frac{d I}{d V} R_{s}\right)\left[G_{p}+\frac{I_{0}}{n v_{t h}} \exp \left(\frac{I R_{S}+V}{n v_{t h}}\right)\right]
$$

Solving (8) for $d I / d V$ and rearranging yields $r(V, I)$ :

$$
r(V, I)=\frac{1}{d I / d V}=\frac{d V}{d I}=-R_{s}-\frac{n v_{t h}}{G_{p} n v_{t h}+I_{0} \exp \left(\frac{I R_{S}+V}{n v_{t h}}\right)} .
$$

Substitution of $R s=0$ and $G p=0$ into (9) yields the dynamic resistance $r i$ of an ideal photovoltaic cell:

$$
r_{i}(V)=-\frac{n v_{t h}}{I_{0} \exp \left(\frac{V}{n v_{t h}}\right)}
$$

Another expression for the dynamic resistance $r(V, I)$ as a function of both terminal variables can be obtained by substitution of (1) into (9):

$$
r(V, I)=\frac{d V}{d I}=-R_{S}-\frac{n v_{t h}}{G_{p}\left(n v_{t h}-V\right)+I_{p h}+I_{0}-I\left(G_{p} R_{S}+1\right)} .
$$

Substitution of $R s=0$ and $G p=0$ into (10) produces another form of dynamic resistance, $r i$, of an ideal cell equivalent to (9i):

$$
r_{i}(I)=-\frac{n v_{t h}}{I_{0}+I_{p h}-I}
$$

\section{B. Dynamic resistance dependence on incident light intensity}

The most important general conclusion that can be drawn from Fig. 3 is that the magnitude of the dynamic resistance $r$, at any voltage in the power-generating quadrant, always decreases as incident light intensity increases. Another conclusion from Fig. 3 worth of analysis is that for any incident light intensity, $r$ decreases as voltage increases from the short-circuit point $(V=0)$ to the open-circuit point $(V=V o c)$. Furthermore, although it might not be fully obvious from Fig. 3, because of the insufficient variation of light intensities used (not wide enough range of values of $I p h$ ), it seems to suggest that the high and low limiting values of $r$ at any voltage are approximately given by $R p+R s$ and $R s$. This behavior of the dynamic resistance, $r$, with incident light intensity can be more conveniently visualized by examining the magnitude of $r$ at the open-circuit point and at the short-circuit points of the $I-V$ curves as a function of photo-generated current Iph.

\section{Dynamic resistance at the open-circuit point}

To calculate the magnitude of the dynamic resistance at the open-circuit point, we evaluate (9) at $V=V o c$ and $I=0$ :

$$
r_{o c}\left(V_{o c}\right)=-R_{S}-\frac{n v_{t h}}{G_{p} n v_{t h}+I_{0} \exp \left(\frac{V_{o c}}{n v_{t h}}\right)} .
$$

For the case of an ideal cell, evaluation of (9i) at $V=V o c$, or substituting $R s=0$ and $G p=0$ into (11) yields:

$$
r_{i o c}\left(V_{o c}\right)=-\frac{n v_{t h}}{I_{0} \exp \left(\frac{V_{o c}}{n v_{t h}}\right)}
$$

Alternatively, evaluating (10) at $V=V o c$ and $I=0$ yields an expression equivalent to (11):

$$
r_{o c}\left(V_{o c}, I_{p h}\right)=-R_{s}-\frac{n v_{t h}}{G_{p}\left(n v_{t h}-V_{o c}\right)+I_{p h}+I_{0}} .
$$

For the case of an ideal cell, evaluation of (10i) at $I=0$, or substitution of $R s=0$ and $G p=0$ into (12) yields an expression equivalent to (11i):

$$
r_{i o c}\left(I_{p h}\right)=-\frac{n v_{t h}}{I_{p h}+I_{0}}
$$

Table II presents three values of $\mid$ rioc $\mid$ and $\mid$ roc $\mid$ as calculated with (12i) and (12), respectively, for three levels of incident light intensity, represented by the given three values of Iph.

The variation of the dynamic resistance's magnitude, roc, calculated with (11) or (12) at the open-circuit point ( $V=V o c$, $I=0)$, is presented in Fig. 4 as a function of photo-generated current intensity, Iph, (which is a direct consequence of the incident light intensity), for $R p=100 \Omega$ and three values of series resistance: $R s=1,4$, and $10 \Omega$.

Fig. 4 indicates that as Iph becomes high (e.g. Iph > 0.04A in this example), the curves start to flatten, tending the magnitude of roc to values near those of $R s(\approx 1,4$, and $10 \Omega$ in this example). This observation is consistent with Fig. 3, which shows the case for $R s=10 \Omega$, where we see that as $V \rightarrow V o c \approx 0.5 \mathrm{~V}$ (open-circuit point) all three curves of $r$ (calculated at three values of $I p h=0.02,0.03$, and 0.04A) approach the value of $R s$ $=10 \Omega$.

Fig. 4 also reveals that the magnitudes of roc increase as Iph decreases until they saturate to constant values around $R p+R s$ $(\approx 101,104$, and $110 \Omega$ in the present example), as Iph reaches very low values (about an order of magnitude lower in the present example). Notice that the saturation condition (roc $\approx R p$ $+R s \approx 110 \Omega$ in this example) cannot be observed at the opencircuit point of curves shown in Fig.3, since none of those three roc curves correspond to a low enough value of Iph. The lowest value of Iph in Fig. 3 is 0.02A, and according to the curve for $R s=10 \Omega$ in Fig. 4, the value of $r o c$ at $I p h=0.02 \mathrm{~A}$ is only slightly larger than $R s$, still far from being able to reach saturation with $r O C \approx R p+R s$.

TABLE II

MAGNITUDE OF THE DYNAMIC RESISTANCE AT OPEN-CIRCUIT CONDITIONS $(V=V O C, I=0)$ OF THE HYPOTHETICAL IDEAL AND REAL SOLAR CELLS, FOR THREE VALUES OF INCIDENT LIGHT INTENSITY

\begin{tabular}{ccc}
\hline \hline $\begin{array}{c}\text { Incident light intensity, } \\
\text { expressed as } \\
\text { photo-current Iph (mA) }\end{array}$ & $\begin{array}{c}\text { Ideal } \mid \text { rioc } \mid(\Omega) \\
G P=0, R S=0\end{array}$ & $\begin{array}{c}\text { Real } \mid \text { roc } \mid(\Omega) \\
R P=100 \Omega, R S=10 \Omega\end{array}$ \\
\hline 20 & 1.939 & 12.462 \\
30 & 1.293 & 11.517 \\
40 & 0.970 & 11.095 \\
\hline \hline
\end{tabular}

Equivalent circuit model diode element's parameters: $v_{t h}=0,02586 \mathrm{~V}, n=1.5, I_{0}=10^{-7} \mathrm{~A}$. 

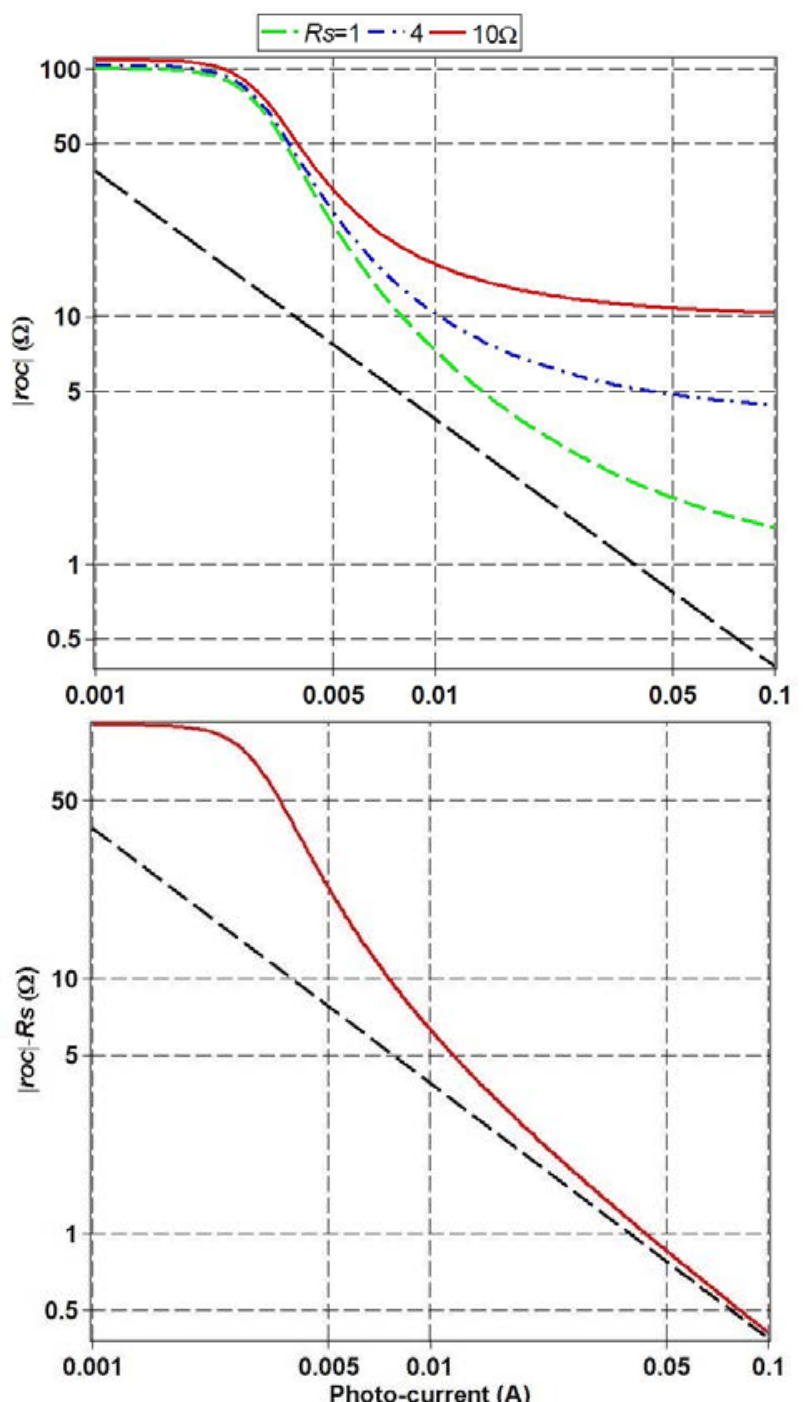

Fig. 4. Log-log plot of the magnitude of the dynamic resistance $|r o c|$, calculated with (11) or (12) at the open-circuit point as a function of photocurrent Iph (upper plot), and the value of $|r o c|$ - Rs (lower plot), for three values of $R$ and $R p=100 \Omega$. Also shown for comparison as a black dash straight line is the magnitude of the dynamic resistance $|r i o c|$ for the corresponding ideal cell $(G p=0$ and $R s=0)$ as calculated with (12i).

The three curves of $|r o c|$ shown in the upper pane of Fig. 4 for different vales of $R s$, may be made to collapse into a single curve by subtracting from $|r o c|$ the corresponding value of $R s$, as (11) and (12) suggest, and the lower pane of Fig.4 illustrates.

We do not present plots of $|r o c|$ vs Isc because the difference with the plots of $|r o c|$ vs Iph shown in Fig. 4 would be almost imperceptible, since only the horizontal axis would need to be shifted by the small difference between Isc and Iph given by (4). In the case of the ideal cell that difference does not exist since (4i) compels Iisc $=I p h$. Instead, we present in Fig. 5 the magnitude of the dynamic resistance $|r o c|$ at the open-circuit point, as a function of the reciprocal short-circuit current $1 / I s c$, calculated with (11) or (12) for the same three values of $R s$ and $R p$. The value of Isc is calculated using (4). Also shown in Fig. 5 for comparison is the $\mid$ rioc $\mid$ vs $1 /$ Iisc of the ideal cell $(\mathrm{Gp}=0$ and $\mathrm{Rs}=0$ ), calculated with (11i) or (12i), with the value of Iisc $=$ Iph according to (4i).

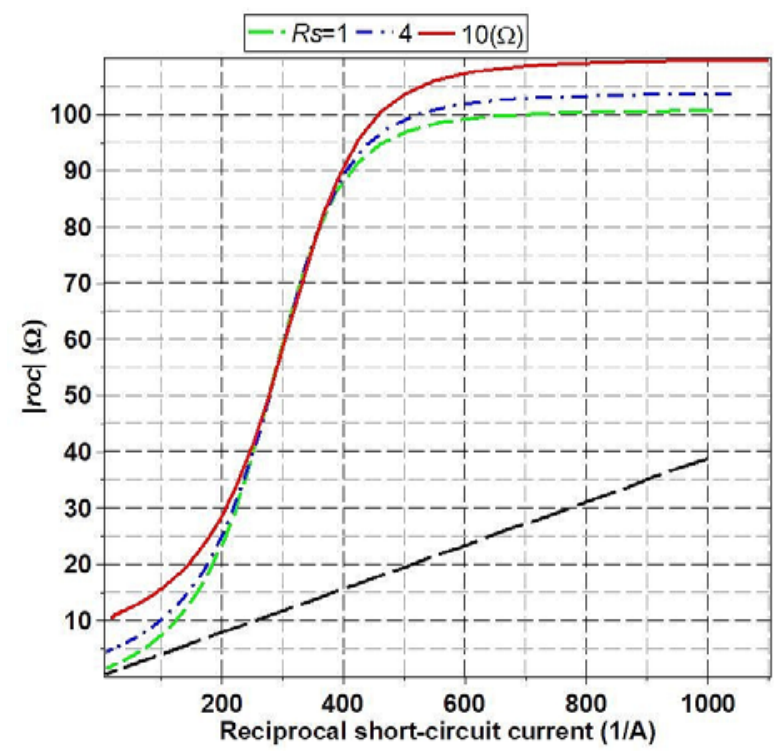

Fig. 5. Linear plots of the magnitude of the dynamic resistance $|r o c|$ as a function of reciprocal short-circuit current $1 /$ Isc, calculated at the open-circuit point with (11) or (12) for three values of $R s$ and $R p=100 \Omega$. The value of $1 / I s c$ is calculated from Iph using (4). Also shown as a black dash straight line, is the $\mid$ rioc $\mid$ of the ideal cell ( $G p=0$ and $R s=0)$ vs 1/Iisc, calculated with (11i) or (12i), using Iisc=Iph according (4i).

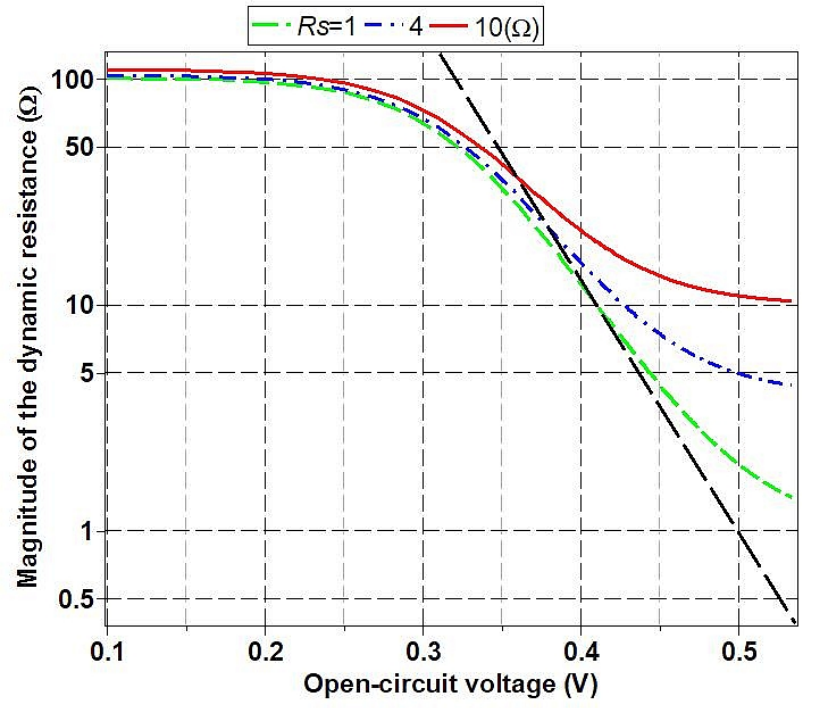

Fig. 6. Semi-logarithmic plot of the magnitude of the dynamic resistance roc at the open-circuit point, as a function of open-circuit voltage, Voc, corresponding to increasing values of Iph, calculated using (11) or (12) for three values of $R s$ and $R p=100 \Omega$. The value of $V o c$ is calculated using (5). Also shown for comparison as a black dash straight line is the dynamic resistance $|r i o c|$ of the ideal cell ( $G p=0$ and $R s=0)$, calculated with (11i).

The variation of the dynamic resistance's magnitude, $|r o c|$, at the open-circuit point is shown in Fig. 6 also as a function of open-circuit voltage, $V o c$, whose value according to (5) corresponds to increasing levels of incident light intensity. It is calculated using (11) with $R p=100 \Omega$ at the three values of $R s=1$, 4, and $10 \Omega$. All three curves shown in Fig. 6 are seen to saturate to a value of $\approx R p+R s$ at low $V o c$ (very low levels of incident light intensity), and to a value of $\approx R s$ at high $V o c$ (very high levels of incident light intensity). Also shown in Fig. 6 for comparison is the magnitude of the dynamic resistance $\mid$ rioc $\mid$ of the corresponding ideal cell ( $G p=0$ and $R s=0$ ), calculated with 
(11i). It is easy to visualize in Fig. 6 how all three curves of $\mid$ roc $\mid$ with $G p \neq 0$ and $R s \neq 0$ would transform into the single straight line representing $\mid$ rioc $\mid$ if their resistive losses $G p \rightarrow 0$ and $R s \rightarrow 0$.

The semi-logarithmic nature of Fig. 6 indicates that since the plot of the magnitude of the ideal cell's $\mid$ rioc $\mid$ as a function of Vioc shows up as a straight line with negative slope, the value of $\mid$ rioc| must vary (decrease) as an exponential function of negative open-circuit voltage, Vioc, which in fact does according to (11i).

On the other hand, Fig. 6 also indicates that, in the case of a real cell ( $G p \neq 0$ and $R s \neq 0$ ), the dependence of $|r o c|$ on Voc happens to be in general considerably different from such purely exponential behavior. How much it differs depends on how significant the magnitudes of $R s$ and $G p$ are, as clearly implied by (11), and illustrated in Fig. 6 by way of the observed saturations to $\mid$ roc $\mid \rightarrow R p+R s$ at low $V o c$ and to $|r o c| \rightarrow R s$ at high $V o c$. Obviously, the smaller the values of $R s$ and $G p$ are, the nearer (11) becomes to (11i), and thus, the closer the behavior of $|r o c|$ would be to that of $\mid$ rioc $\mid$, which behaves as a decreasing function of the open-circuit voltage.

\section{Dynamic resistance at the sort-circuit point}

Let us now look at the other end of the power-generating quadrant, i.e., the short-circuit point, defined by the coordinates $(V=0, I=I s c)$. The magnitude of the dynamic resistance $|r s c|$ at the sort-circuit point is also a useful parameter to look at. To calculate it we need to evaluate (9) at $V=0$ and $I=I s c$ :

$$
r_{s c}\left(I_{s c}\right)=-R_{s}-\frac{n v_{t h}}{G_{p} n v_{t h}+I_{0} \exp \left(\frac{I_{s c} R_{s}}{n v_{t h}}\right)} .
$$

In the case of an ideal cell, substitution of $R s=0$ and $G p=0$ into (13) yields a constant value of $\mid$ risc $\mid$ :

$$
r_{i s c}=-\frac{n v_{t h}}{I_{0}},
$$

Alternatively, evaluation of (10) at $V=0$ and $I=I s c$ yields an expression equivalent to (13):

$$
r_{s c}=r\left(I_{p h}, I_{s c}\right)=-R_{s}-\frac{n v_{t h}}{G_{p} n v_{t h}-I_{s c}\left(G_{p} R_{S}+1\right)+I_{p h}+I_{0}},
$$

For the case of an ideal cell, substitution of $R s=0$ and $G p=0$ into (14) yields the same constant as in (13i):

$$
r_{i s c}=-\frac{n v_{t h}}{I_{0}} .
$$

The light intensity Iph dependence of the magnitude of dynamic resistance $|r s c|$ calculated at the sort-circuit point is presented in Fig. 7, for the same values of $R p=100 \Omega$ and $R s=1$, 4 , and $10 \Omega$. A quick look reveals that $|r s c|$ is clearly a function of light intensity Iph, in a way that in general qualitatively resembles that of $\mid$ roc $\mid$ shown in Fig.4. But this similarity refers only the fact that when Iph reaches very large values, $|r s c|$ exhibits saturated values near those of $R s(\approx 1,4$, and $10 \Omega$ in this example); and that as the value of Iph starts to decrease, $|r s c|$ starts to increase until at low values of $I p h,|r s c|$ saturates to constant values around those of $R p+R s(\approx 101,104$, and $110 \Omega$ in the present example).

Table III presents values of $|r s c|$ and $|r i s c|$ for three values of Iph. Notice that in the case of the ideal cell, the value of $|r i s c|$ is very large and constant at any incident light intensity (represented by Iph).

Fig. 7 is perfectly compatible with the behavior observed in Fig. 3. Notice, e.g., that when $I p h=0.02 \mathrm{~A}$, the $r s c$ curve corresponding to $R s=10 \Omega$ (solid red line in Fig. 7) reaches a constant value of $\approx R p+R s(\approx 107 \Omega$ in this example). This is the same value that the $|r|$ curve that corresponds to $I p h=0.02 \mathrm{~A}$ (dashed green line in Fig.3) reaches when $V \rightarrow 0$ (short-circuit point) in Fig. 3.

\section{DISCUSION}

We have just established that the magnitudes of the dynamic resistance at the short-circuit and the open-circuit points, $|r s c|$ and $|r s c|$, both experience a transition when Iph changes as a consequence of a change of the incident light intensity. They both decrease from $\sim R p+R s$ at very low incident light intensities (very low Iph) to $\sim R s$ at very high incident light

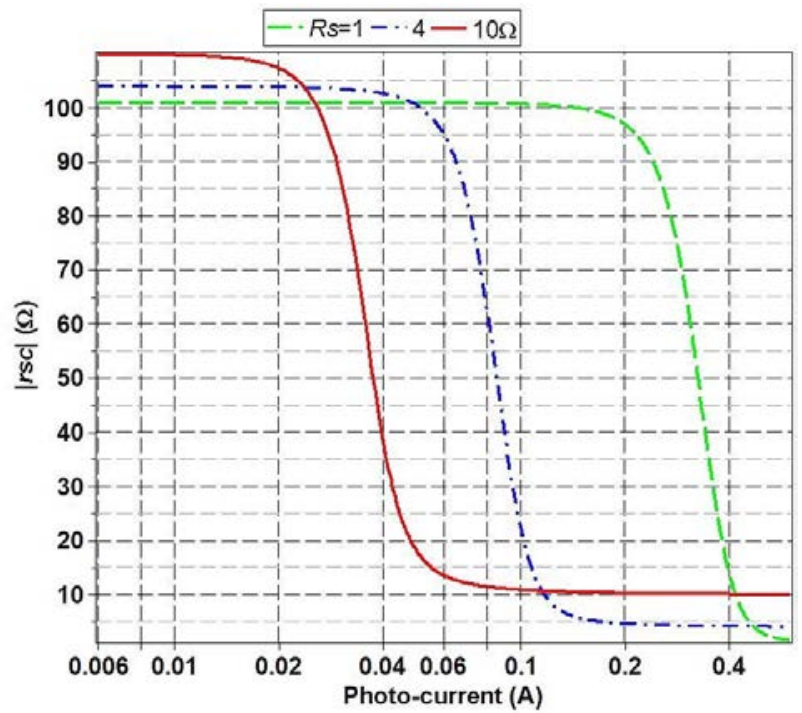

Fig. 7. Semi-logarithmic plot of the magnitude of the dynamic resistance $r s c$ calculated at the short-circuit point $(V=0, I=I s c)$ on the $I-V$ characteristics of the illuminated hypothetical cell, as a function of photo-current Iph, for $R p=100 \Omega$ and three values of $R s$. The plot of the dynamic resistance |risc| of the ideal cell ( $G p=0$ and $R s=0$ ), calculated with (13i), may not be included here for comparison, since it is a very large constant (see Table III) that falls outside the bounds of this figure.

TABLE III

MAGNITUDE OF THE DYNAMIC RESISTANCE AT SHORT-CIRCUIT CONDITIONS $(V=0, I=I S C)$ OF THE HYPOTHETICAL IDEAL AND REAL SOLAR CELL, FOR THREE VALUES OF INCIDENT LIGHT INTENSITY

\begin{tabular}{ccc}
\hline $\begin{array}{c}\text { Incident light intensity, } \\
\text { expressed as } \\
\text { photo-current Iph }(\mathrm{mA})\end{array}$ & $\begin{array}{c}\text { Ideal } \mid \text { risc| }(\mathrm{k} \Omega) \\
G P=0, R S=0\end{array}$ & $\begin{array}{c}\text { Real }|r s c|(\Omega) \\
R p=100 \Omega, R s=10 \Omega\end{array}$ \\
\hline 20 & 387.9 & 107.28 \\
30 & 387.9 & 87.87 \\
40 & 387.9 & 39.11 \\
\hline \hline
\end{tabular}

Equivalent circuit model diode element's parameters:

$v_{t h}=0,02586 \mathrm{~V}, n=1.5, I_{0}=10^{-7} \mathrm{~A}$. 
intensities (very high Iph). Although the observed behavior of these two magnitudes appear to follow similar sigmoid-function type of behavior, a more careful comparison of Figs. 4 and 7 clearly reveals that their transitions from high to low values as the light intensity increases differ from each other. Several differences can be visualized in the present example. The first observation is that the transition of $|r o c|$ (Fig. 4) occurs at lower values of Iph than the transition of $|r s c|$ (Fig. 7), and this is so for all values of $R$.

The second observation is that a similar change of the value of Rs causes a much larger shift on the Iph axis (wider spread) of the transition of $|r s c|$ (Fig. 7) than that of $|r o c|$ (Fig. 4). The shift produced by increasing the value of $R s$ by the same amount is smaller in the case of $|r o c|$ (Fig. 4) than in the case of $|r s c|$ (Fig. 7).

The third and most obvious observation refers to how the magnitudes of $|r o c|$ and $|r s c|$ vary in different ways. In the case of $|r s c|$ that difference is a consequence of the crossovers of the curves, which can mean a total reversal of the dependence direction, as can be seen in the central region of Fig.7. For example, assuming a value of $I p h$ of $0.08 \mathrm{~A}$, the curves presented in Fig. 7 for $|r s c|$ indicate that if the value $R s$ increases from 1 , to 4 , and then to $10 \Omega$, the corresponding magnitude of $|r s c|$ experiences a direct reduction from $\sim 101$, to $\sim 62$, and then to $\sim 12 \Omega$. This is the opposite dependence that occurs at very high and low values of Iph, shown at the right and left sides, respectively, of Fig. 7. Even stranger behavior of $|r s c|$ can be expected when $R s$ changes at certain values of Iph, e.g. at $I p h=0.2 \mathrm{~A}$ in Fig. 7. This type of effect does not exist in the case of $|r o c|$, whose value always increases as Rs increases at any value of Iph, as shown Fig. 4.

A common mistake is falling to the temptation of a priori assuming that $I p h \approx I s c$ as a good approximation regardless of any considerations about the presence of significant parasitic resistive losses; an assumption that is in general disavowed by (4). Another approximation mistake that unfortunately is often made about the dynamic resistance of illuminated solar cells is to naively assume that is always approximately equal to $R s$ when measured at the open-circuit point, or on the end of the power-producing quadrant, assuming that the dynamic resistance measured at the short-circuit point is always approximately equal to $R p$ [6]. While these two approximations could be perfectly assumable in many instances, the hazard is, as we have shown here, to assume that these approximations are always justified, regardless of the magnitude of parasitic resistive losses and the intensity of the incident light, as Figs. 4 and 7 clearly indicate. The results of this analysis, therefore, call attention to the need to handle these assumptions about the dynamic resistance with care, always checking their validity before proceeding to draw conclusions that depend on them.

\section{CONCLUSIONS}

The following conclusions may be drawn about the expected theoretical behavior of the dynamic resistance of illuminated solar cells:

1) In general, the magnitude of the dynamic resistance of an illuminated photovoltaic cell measured (or calculated) at any point of its static $I-V$ characteristic, depends on the illumination intensity incident upon the cell's surface. This includes, of course, the dynamic resistance measured at the points of the $I-V$ curve corresponding to short circuit, open circuit, and MPP conditions. The incident light dependence of the dynamic resistance, therefore, can affect the ability to effectively track the MPP of a solar cell or panel as the intensity of the solar illumination changes hourly, or because of cloud shadowing, during the course of the day.

2) If the intensity of incoming illumination is allowed to increase, e.g. through light concentration, so that the photocurrent can reach high enough levels, the value of the dynamic resistance can eventually become approximately equal to the value of the lumped series resistive loss $(R s)$ at any operating point along the $I-V$ characteristic, including the short-circuit, the open-circuit, and the MPP points.

3) Likewise, if the level of illumination decreases drastically, e.g. at night or under cloud cover in the case of terrestrial solar cells, so that the intensity of the photocurrent becomes low enough, the value of the dynamic resistance will approach and eventually will saturate to a value approximately equal to the sum of lumped series and parallel resistive loses $(R s+R p)$ at any operating point along the $I-V$ characteristic, including the short-circuit, the opencircuit, and the MPP points.

4) Although a trivial fact, it is important to keep in mind that changing the illumination level might significantly change the values of both Isc and Voc, which behave as linear and logarithmic functions of the photo-generated current, respectively.

\section{ACKNOWLEDGMENT}

Helpful discussions with countless unnamed students and colleagues have contributed over time to better understand the complex implications of this issue.

\section{REFERENCES}

[1] A. Ortiz-Conde, F. J. García-Sánchez, J. Muci, A. Sucre-González, “A review of diode and solar cell equivalent circuit model lumped parameter extraction procedures," Facta Universitatis, Series: Electronics and Energetics, vol. 27, no. 1, pp. 57-102, March 2014. http://dx.doi.org/10.2298/FUEE1401057O

[2] J. Thongpron, K. Kirtikara, C. Jivacate. "A method for the determination of dynamic resistance of photovoltaic modules under illumination." Solar Energy Materials and Solar Cells, vol. 90, no. 18-19, pp, 3078-3084, Nov. 2006. https://doi.org/10.1016/j.solmat.2006.06.029

[3] W. Shockley, "The theory of p-n junction in semiconductors and p-n junction transistors," Bell Syst Tech J., vol. 28, pp. 435-489, 1949. https://doi.org/10.1002/j.1538-7305.1949.tb03645.x

[4] R. M. Corless, G. H. Gonnet, D. E. G. Hare, D. J. Jeffrey, D. E. Knuth, "On the Lambert W Function," Adv Comput Math, vol. 5, no. 1, pp. 329 - 359, Dec. 1996. https://doi.org/10.1007/BF02124750

[5] A. Jain, A, Kapoor, "Exact analytical solutions of the parameters of real solar cells using Lambert W-function," Solar Energy Materials and Solar Cells, vol. 81, no. 2, pp. 269-277, 6 Feb 2004. https://doi.org/10.1016/j.solmat.2003.11.018

[6] K. Tada, "Calculation of error in series/shunt resistance estimated from current-voltage slope using exact analytical expressions with Roberts gfunction." IEEJ Trans. Electrical \& Electronic Engineering, vol. 14, no. 2, pp. 333-334, Feb. 2019. https://doi.org/10.1002/tee.22813 